DOI: $10.4274 /$ jarem.galenos.2020.2556

J Acad Res Med 2020;10(2):175-9

\title{
A Comparison of Exercise Tolerance, Measures of Cardiac Response to Exercise and Serum Markers in Chronic Obstructive Pulmonary Disease
}

\author{
(D) Pinar Mutlu, (D) N. Arzu Mirici \\ Çanakkale Onsekiz Mart University Faculty of Medicine, Department of Chest Diseases, Çanakkale, Turkey
}

Cite this article as: Mutlu P, Mirici N.A. A Comparison of Exercise Tolerance, Measures of Cardiac Response to Exercise and Serum Markers in Chronic Obstructive Pulmonary Disease. J Acad Res Med 2020;10(2):175-9

\begin{abstract}
Objective: This study investigates the relationship between cardiac response measures and serum markers with exercise performance in patients with chronic obstructive pulmonary disease (COPD).

Methods: A total of 90 patients with stable COPD, diagnosed based on clinical findings and the GOLD spirometric criteria were included in this study. Each patient completed pulmonary function and lung diffusion tests, a six-minute walking test (6MWT), a Modified Medical Research Council Dyspnea scale, and a St. George Respiratory Questionnaire. The patient's height and body weight were obtained, and the body mass index was calculated. Serum pro-brain natriuretic peptide (pro-BNP), tumor necrosis factor- $\alpha$, interleukin-6 (IL-6), IL-8, transferrin, and C-reactive protein levels were measured.

Results: After the evaluation of exercise tolerance with a $6 \mathrm{MWT}$, exercise performance was found to be significantly associated with spirometric measurements, the level of dyspnea, quality of life, and serum pro-BNP levels. When the measures of cardiac response to exercise were evaluated, significant relationships were noted between systolic pressure and pulse differences, and the distance walked in a 6MWT.

Conclusion: We believe that the many factors that determine exercise tolerance in COPD patients are interrelated. A clear demonstration of these relationships may, in the long-term, change the perspective of both the clinical course of the disease and its treatment strategies.

Keywords: COPD, Pro-BNP, transferrin, exercise performance, 6-minutes walking test
\end{abstract}

\section{INTRODUCTION}

Chronic obstructive pulmonary disease (COPD) is a slowdeveloping, chronic, and progressive disorder of the respiratory system. Treatment is based on the patient's symptoms, given that there is currently no treatment that may prevent the development or cure of COPD (1). Dyspnea is the most common symptom seen in COPD and is the leading cause of functional loss. It, therefore, plays an essential role in the choice of medication for COPD. The perceived level of dyspnea is considered as a marker of the patient's quality of life (2).

Musculoskeletal dysfunction and weakness are among the most common systemic effects of COPD and are frequently accompanied by a loss of fat-free mass. The loss of muscle mass is accompanied by muscle weakness, and this is a significant predictor of exercise capacity in COPD patients, irrespective of disease severity (3-5).

ORCID IDs of the authors: P.M. 0000-0002-7496-0026; N.A.M. 0000-0002-7189-9258. 
In COPD patients, increased levels of tumor necrosis factor (TNF- $\alpha$ ), interleukin 6 (IL-6), and IL-8, and decreased levels of calcium in the mitochondria may induce apoptosis in all body cells, including muscle cells, leading to a decreased body mass index (BMI) and weight loss $(6,7)$.

Pro-B-type natriuretic peptide (Pro-BNP) is synthesized in response to a decrease in ventricular myocytes (8), and plasma pro-BNP levels are elevated in COPD patients that developed pulmonary hypertension and right ventricular overload (9). There are no studies on pro-BNP levels in stable COPD patients with no cor pulmonale or pulmonary hypertension (10).

A six-minute walking test (6MWT) is a valid and reliable test for the objective measurement of functional exercise capacity in individuals with pulmonary or cardiovascular diseases. The test is simple, reproducible, inexpensive, and well-tolerated. It better reflects the ability of a patient to engage in daily life activities compared to other walking tests (11-14). In this study, we compare the functional exercise capacity of COPD patients based on a 6MWT with measurements of cardiac response to exercise and serum markers.

\section{METHODS}

Patient selection: The study population included 90 patients with stable COPD, including 82 men and 8 women, selected from among the patients who were referred to our Clinics of Pulmonology Outpatient between February 2010 and April 2012, and who were invited to take part in this study through face-toface interviews or telephone contacts.

A written informed consent was obtained from each patient. Ethics committee approval was received from the University Clinical Research Ethics Committee for approval of the study (approval number: 2010.03, date: 15.01.2010). Written permission was obtained from University Medical School Dean's Office in order to conduct the study in the Chest Diseases Clinic of Research and Application Hospital. The study was conducted in accordance with the principles of the Declaration of Helsinki.

Study inclusion criteria: Patients with a clinical picture consistent with COPD, diagnosed based on the GOLD spirometric criteria, and stable disease (no COPD exacerbation within the last six weeks) were included in this study.

Study exclusion criteria: Patients with severe physical disabilities who were unable to take the 6MWT, myocardial infarction or unstable angina within the last one month, or a history of heart failure were excluded from this study.

Our work conforms to the Helsinki declaration. This study was approved by the local research ethics committee, and all patients were informed about this study and provided written informed consent for their participation. The patients underwent physical examinations and clinical evaluations. To measure the severity of dyspnea and identify the COPD stage, all patients performed pulmonary function and lung diffusion tests and were assessed according to the Modified
Medical Research Council (mMRC) dyspnea scale. Exercise performance was evaluated by a 6MWT. Height and body weight were obtained and BMI was calculated.

Pulmonary function test: This was performed by a pulmonary function test nurse in the pulmonary function test laboratories of our clinics using a Masterscope JLAB V5.22.1.50 (Cardinal Health, Germany, Hoechberg, 2006) spirometry device.

Lung diffusion test: This was carried out using a Vmax Encore 229 (Viasys Respiratory Care, ABD, California, 2008) device, based on the single-breath diffusing capacity of lung for carbon monoxide (DLCO) method.

Modified Medical Research Council Dyspnea scale: This scale is used to measure the shortness of breath that develops during walking or working, and differentiates five stages.

Six-minute walking test: This test was used to measure exercise capacity based on the recommendations of the American Thoracic Society. The total distance walked was measured and recorded at the end of the test.

St. George Respiratory Questionnaire: This is a selfadministered questionnaire, standardized specifically for airway disorders, and includes questions related to three aspects of the disorder, including symptoms (8 questions), activity (16 questions) and impact (26 questions) (14).

Blood samples: TNF- $\alpha$, IL-6, and IL-8 levels were measured twice using a high-precision enzyme-linked immunosorbent assay (Biosource, Nivelles, Belgium). C-reactive protein (CRP) levels were measured using a latex-enhanced immunonephelometry (Siemens, Dublin, Ireland). Plasma pro-BNP levels were measured through an electrochemiluminescence immunoassay using an Elecsys 2010 system (Roche Diagnostics, Mannheim, Germany). Transferrin levels were measured using the ELISA method.

Skinfold thickness measurement: Triceps measurements were obtained vertically from the midpoint of the distance between the acromion and olecranon while the arms were left to rest freely on either side of the body.

\section{Statistical Analysis}

The data were analyzed using SPSS Package Software version 15.0 (IBM Corporation, Armonk, New York, USA). Descriptive data were presented as numbers, percentages, means, standard deviations, medians, and minimum-maximum values. Categorical data were analyzed with a chi-square test, while a Kruskal-Wallis test was used to compare continuous variables. Correlation analyses were carried out with Spearman, Pearson, and Kendall's Tau tests, and correlation power values were interpreted as follows: 0.00-0.24, weak; 0.25-0.49, moderate; 0.50-0.74, strong; and 0.75-1.00, very strong. $\mathrm{P}<0.05$ were considered statistically significant.

\section{RESULTS}

There were 82 men and 8 women included in this study, with a mean age of $59.7 \pm 5.8$ years. Most patients were in the GOLDstage 2 (64.4\%) and mMRC 2 (55.6\%) groups (Table 1). 
When measuring exercise tolerance using the 6MWT, the minimum and maximum walking distances were 180 and 671 meters, respectively, with a mean walking distance of $494.16 \pm 88.57$ meters.

Based on the GOLD spirometric staging, the relationship between the St. George Respiratory Questionnaire (SGRQ) scores and the total distance walked in the 6MWT was examined, using Spearman's correlation test (Table 2).

In patients with stage 1 COPD (FEV1 $\geq 80 \%$ ), a significant negative correlation was identified between the SGRQ symptom score and the total distance walked in 6MWT (rho: $-0.611, p=0.009$ ), and the SGRQ total score was found to have a significant negative but weak correlation with the 6MWT distance (rho: -0.493, $p=0.044)$.

In patients with stage 2 COPD (FEV1 51\%-79\%), the SGRO activity, impact, and total scores had significant negative but weak correlations with total distance walked in the 6MWT (for

\begin{tabular}{|l|l|}
\hline \multicolumn{2}{|l|}{ Table 1. GOLD and mMRC stages of patients } \\
\hline Stage & Patient (n, \%) \\
\hline GOLD 1 & $17(18.9)$ \\
\hline GOLD 2 & $58(64.4)$ \\
\hline GOLD 3 & $14(15.6)$ \\
\hline GOLD 4 & $1(1.1)$ \\
\hline mMRC 1 & $19(21.1)$ \\
\hline mMRC 2 & $50(55.6)$ \\
\hline mMRC 3 & $18(20)$ \\
\hline mMRC 4 & $3(3.3)$ \\
\hline GOLD: mMRC: Modified medical research council
\end{tabular}

Table 2. Relationship between total walking distance and COPD stage based on GOLD spirometric measurement criteria (Spearman correlation) (rho: correlation coefficient)

\begin{tabular}{|c|c|c|c|}
\hline Stage & \multirow{2}{*}{$\begin{array}{l}\text { SGRQ score } \\
\text { Symptom score }\end{array}$} & \multicolumn{2}{|c|}{ Total distance } \\
\hline \multirow{4}{*}{$\begin{array}{l}\text { Stage } 1 \\
\text { (FEV1 \% } 280 \text { ) }\end{array}$} & & $p=0.009$ & rho $=-0.611$ \\
\hline & Activity score & $p=0.084$ & rho $=-0.431$ \\
\hline & Impact score & $p=0.142$ & rho $=-0.372$ \\
\hline & Total & $p=0.044$ & rho $=-0.493$ \\
\hline \multirow{4}{*}{$\begin{array}{l}\text { Stage } 2 \\
\text { (FEV1 \%51-79) }\end{array}$} & Symptom score & $p=0.063$ & rho $=-0.245$ \\
\hline & Activity score & $p=0.0001$ & rho $=-0.532$ \\
\hline & Impact score & $p=0.004$ & rho $=-0.370$ \\
\hline & Total & $p=0.003$ & rho $=-0.390$ \\
\hline \multirow{4}{*}{$\begin{array}{l}\text { Stage } 3 \\
\text { (FEV1 \%31-50) }\end{array}$} & Symptom score & $p=0.503$ & rho $=-0.196$ \\
\hline & Activity score & $p=0.792$ & rho $=0.078$ \\
\hline & Impact score & $p=0.881$ & rho $=-0.04$ \\
\hline & Total & $p=0.994$ & rho $=-0.002$ \\
\hline
\end{tabular}

the activity score, rho: $-0.532, p=0.0001$; for the impact score, rho: $-0.370, p=0.004$; for the total score, rho: $-0.390, p=0.003)$. Table 3 shows the relationships between the 6MWT distance and other parameters investigated in this study (Table 3).

A significant moderate negative correlation was found between the 6MWT walking distance and pro-BNP levels (rho: -0.435; $p=0.003$ ). Post-test forced vital capacity (FVC) and FEV1 values had a significant moderate positive correlation with walking distance (For FVC, rho: 0.354; $p=0.001$; for FEV1, rho: $0.332 p=0.001$ ). On the other hand, a weak positive correlation was found between the DLCO value and total walking distance (rho: $0.212 ; p=0.048$ ), and a significant weak negative correlation between $\mathrm{BMI}$ and total walking distance ( $r$ ho: $-0.217 ; p=0.039$ ). A moderate negative correlation was found between total distance walked in 6MWT and the degree of dyspnea at the end of the test (rho: -0.451 ; $\mathrm{p} \square 0.01)$.

Values recorded during the 6MWT had a moderate negative correlation with the SGRQ symptom, impact, and total scores (rho: -0.364 , $\mathrm{p} \square 0.001$; rho: -0.384 , $\mathrm{p} \square 0.001$; rho: -0.449 , $\mathrm{p} \square 0.001$, respectively), and a significant strong negative correlation with the SGRQ activity scores (rho: -0.524, p $\square 0.001$ ).

The total walking distance had a moderate negative correlation with the level of dyspnea experienced after the test (rho: -0.311 ;

Table 3. Relationships between 6MWT distance and other investigated parameters

\begin{tabular}{|c|c|c|}
\hline Investigated parameter & Associated-rho & Significance-p \\
\hline Serum Pro-BNP levels & -0.435 & 0.003 \\
\hline Post-test FVC & 0.354 & 0.001 \\
\hline Post-test FEV1 & 0.332 & 0.001 \\
\hline DLCO & 0.212 & 0.048 \\
\hline BMI & -0.217 & 0.039 \\
\hline SGRQ total** & -0.449 & $\square 0.01$ \\
\hline SGRQ-symptoms & -0.364 & $\square 0.01$ \\
\hline SGRQ activity & -0.524 & $\square 0.01$ \\
\hline SGRQ-impact & -0.384 & $\square 0.01$ \\
\hline$M R C^{*}$ & -0.451 & $\square 0.01$ \\
\hline $\begin{array}{l}\text { Level of dyspnea at the end of } \\
\text { the test }\end{array}$ & -0.311 & 0.003 \\
\hline $\begin{array}{l}\text { Before/after the test - Pulse } \\
\text { difference }\end{array}$ & 0.348 & 0.001 \\
\hline $\begin{array}{l}\text { Systolic blood pressure } \\
\text { difference before/after test }\end{array}$ & 0.266 & 0.01 \\
\hline Triceps skinfold thickness ${ }^{\star \star}$ & -0.266 & 0.01 \\
\hline \multicolumn{3}{|c|}{$\begin{array}{l}\text { *The relationship between the total Six-minute Walking test (6MWT) } \\
\text { distance and the medical research council was analyzed with a Kendall's } \\
\text { Tau test, **The relationships between the total 6MWT distance, the St. } \\
\text { George respiratory questionnaire scores, and triceps skinfold thickness were } \\
\text { analyzed with a Spearman's correlation test } \\
\text { Pro-BNP: Brain natriuretic peptide, 6MWT: Six-minute Walking test, MRC: } \\
\text { Medical Research Council, SGRQ: St. George respiratory questionnaire, } \\
\text { FVC: forced vital capacity }\end{array}$} \\
\hline
\end{tabular}


$p=0.003)$, and positive moderate correlations with the pulse and systolic pressure differences recorded after the test (rho: 0.348 , $p=0.001$; rho: $0.266, p=0.01$ ). The relationship between the total walking distance and triceps skinfold thickness, as a marker of nutrition, was investigated. The 6MWT distance had a moderate negative correlation with triceps thickness (rho: $-0.266, p=0.01$ ) and with BMI (rho: $-0.274, p \square 0.01$ ).

\section{DISCUSSION}

In the present study, a 6MWT was used to evaluate the exercise capacity of COPD patients, as an easy-to-implement and practical test, and a correlation was identified between the performance of daily life activities of patients and their 6MWT results (15).

In line with the findings in the literature, we identified a relationship between the 6MWT distances and SGRQ scores of mMRC stage 1 and stage 2 COPD patients, which indicates that the quality of life of COPD patients becomes poorer as their exercise capacity decreases. Three percent of the patients in this study had very severe (stage 4), and $18 \%$ had severe (stage 3) COPD. This insufficient number of patients with severe levels of the disease may represent an important limitation of our study.

While the source of systemic inflammation in COPD has yet to be identified, there have been some suggested mechanisms. Smoking may result in systemic inflammation even in the absence of airway obstruction, and may also cause extrapulmonary conditions $(3,4)$.

Another opinion suggests that inflammation in the lungs is an important source of systemic inflammation. Proinflammatory molecules, such as TNF- $\alpha$ and IL-1, IL-6, IL-8 and transforming growth factor beta, which are released from the inflammatory cells into the lung parenchyma, flow from the lungs and enter into the systemic circulation, leading to the activation of inflammatory cells and eventually causing systemic inflammation $(3,16)$.

In addition to its contribution to the extrapulmonary effects of COPD, the severity of systemic inflammation is also directly associated with poor quality of life, increased airway limitation, and decreased exercise tolerance in COPD patients (3). In our study, we could not find a significant correlation between exercise capacity and inflammatory markers.

Garcia-Rio et al. (17) found out that the exercise tolerance measured using a 6MWT was negatively proportional to serum CRP, IL-6, and IL-8 levels contrary to our results. Several cross-sectional studies have reported significant relationships between plasma CRP levels, disease severity, quality of life, exercise capacity, treatment response, and mortality $(18,19)$. Similar to our findings, however, Gagnon et al. (20) could not find a significant relationship between plasma IL-6 levels and exercise capacity in patients with mild COPD. The relationship between hyperinflation and inflammation is an interesting hypothesis that merits further investigation.

We identified a moderate negative correlation between 6MWT distance and pro-BNP levels. According to Stols's hypothesis,
BNP levels that increase in the presence of COPD as the small pulmonary arteries contract due to hypoxia result in increased pulmonary arterial pressure and cardiac stress (21).

In a study that evaluated cardiopulmonary exercise test (CPET) findings and BNP levels in COPD patients, Eroğlu et al. (22) identified a negative correlation between BNP levels and CPET parameters in patients with dyspnea and isolated left ventricular diastolic dysfunction. They suggested that BNP levels may be a beneficial biomarker of limited exercise capacity in patients with moderate to severe COPD (22).

Gas diffusion is impaired in COPD due to alveolar destruction and vascular bed loss (23). Consistent with the literature, we identified a positive correlation between $6 \mathrm{MWT}$ and $\operatorname{DLCO}(24,25)$.

Dyspnea is the most common symptom among COPD patients. The former is chronic, progressive, and persistent. COPD patients frequently develop dynamic hyperinflation during exercise that limits their daily activities (25). We evaluated the severity of dyspnea using the mMRC scale and identified a correlation between 6MWT and dyspnea severity. This finding is consistent with previous studies in which strong correlations are reported between exercise capacity and dyspnea severity experienced during exercise. (23-25).

We also identified significant correlations between 6MWT distance and pre-/post-test pulse and systolic blood pressure differences. Pulse response during the test is an important parameter regarding cardiac response to exercise, guiding the investigation of heart failure in patients with limited exercise capacity.

We evaluated malnutrition levels based on $\mathrm{BMI}$ and triceps skin thickness. The 6MWT distance of our patients was negatively correlated with BMI and triceps skin thickness. Malnutrition is associated with structural and metabolic changes in the peripheral respiratory muscles, and so may exacerbate shortness of breath and impair exercise tolerance and quality of life (26). While many previous studies have failed to identify a correlation between body weight and 6MWT distance, nutritional support significantly increased the distance walked in 6MWT (27-29).

\section{Study Limitations}

The limitations of the study include the collection of study data from a single center and the small sample size. Also, as this is a cross-sectional study, cause-effect analysis cannot be performed.

\section{CONCLUSION}

The data reported over recent years suggest that exercise performance in COPD patients may be a direct or indirect predictor of survival. While this process involves rather complex associations, it is specific for each patient. In this regard, all factors that reduce exercise capacity should be identified and eliminated to increase the patients' quality of life and survival as much as possible.

The data collected in this study and the relevant analyses suggest that there are numerous and interrelated factors that affect 
12. Puente-Maestu L, Tena T, Trascasa C, Pérez-Parra J, Godoy R, García MJ, et al. Training improves muscle oxidative capacity and oxygenation recovery kinetics in patients with chronic obstructive pulmonary disease. Eur J Appl Physiol 2003; 88: 580-7.

13. Cahalin L, Pappagianopoulos P, Prevost S, Wain J, Ginns L. The relationship of the 6-min walk test to maximal oxygen consumption in transplant candidates with end-stage lung disease. Chest 1995; 108: $452-$ 9.

from the University Clinical Research Ethics Committee for approval of the study (15.01.2010, Approval No. 2010.03). Written permission was obtained from University Medical School Dean's Office in order to conduct the study in the Chest Diseases Clinic of Research and Application Hospital. The study was conducted in accordance with the principles of the Declaration of Helsinki.

Informed Consent: A written informed consent was obtained from each patient.

Peer-review: Externally peer-reviewed.

Author Contributions: Concept - P.M., N.A.M.; Design - P.M., N.A.M.; Data Collection and/or Processing - P.M., N.A.M.; Analysis and/or Interpretation - P.M., N.A.M.; Writing - P.M.; Critical Review - N.A.M.

Conflict of Interest: The authors have no conflict of interest to declare.

Financial Disclosure: The authors declared that this study has received no financial support.

\section{REFERENCES}

1. van Manen JG, Bindels PJE, Dekker FW, lizermans CJ, van der Zee JS, Schadé E. Risk of depression in patients with chronic obstructive pulmonary disease and its determinants. Thorax 2002; 57: 412-6.

2. Hill K, Geist R, Goldstein RS, Lacasse Y. Anxiety and depression in end stage COPD. Eur Respir J 2008; 31: 667-77.

3. Umut S, Saryal S. Turkish thoracic society chronic obstructive lung disease diagnosis and treatment consensus report. Turkish Thoracic Journal 2010; 11: 26-30

4. Sevenoaks MJ, Stockley RA. Chronic obstructive pulmonary disease, inflammation and comorbidity- a common inflammatory phenotype? Respir Res 2006; 7: 70

5. Global Strategy for the Diagnosis, Management and Prevention of Chronic Obstructive Pulmonary Disease GOLD Report Revised 2011: p.48-74.

6. Agusti A, Soriano JB. COPD As A Systemic Disease. COPD 2008; 5: $133-$

7. Gosker HR, Kubat B, Schaart G, van der Vusse GJ, Wouters EF, Schols AM. Myopathological features in skeletal muscle of patients with chronic obstructive pulmonary disease. Eur Respir J 2003; 22: 280-5.

8. Inoue $\mathrm{Y}$, Kawayama T, Iwanaga T, Aizawa H. High plasma brain natriuretic peptide levels in stable COPD without pulmonary hypertension or cor pulmonale. Intern Med 2009; 48: 503-12

9. Bando M, Ishii Y, Sugiyama Y, Kitamura S. Elevated plasma brain natriuretic peptide levels in chronic respiratory failure with cor pulmonale. Respir Med 1999; 93: 507-14

10. Leuchte HH, Holzapfel M, Baumgartner RA, Ding I, Neurohr C, Vogeser $\mathrm{M}$, et al. Clinical significance of brain natriuretic peptide in primary pulmonary hypertension. J Am Coll Cardiol 2004; 43: 764-70.

11. Gosker HR, Bast A, Haenen GRMM, Fischer MAJG, van der Vusse GJ, Wouters EFM, et al. Altered antioxidant status in peripheral skeletal muscle of patients with COPD. Respir Med 2005; 99: 118-25.
14. Solway S, Brooks D, Lacasse $Y$, Thomas S. A qualitative systematic overview of the measurement properties of functional walk tests used in the cardiorespiratory domain. Chest 2001; 119: 256-70.

15. Light RW, Merrill EJ, Despar JA, Gordon GH, Mutalipassi LR. Prevalence of depression and anxiety in patients with COPD. Relationship to functional capacity. Chest 1985; 87: 35-8.

16. Barnes PJ, Celli BR. Systemic manifestations and comorbidities of COPD. Eur Respir J 2009; 33: 1165-85.

17. Garcia-Rio F, Miravitlles M, Soriano JB, Muñoz L, Duran-Tauleria E, Sánchez G; EPI-SCAN Steering Committee, et al. Systemic inflammation in chronic obstructive pulmonary disease: a population-based study. Respir Res 2010, 11: 63.

18. Dahl M, Vestbo J, Lange P, Bojesen SE, Tybjaerg-Hansen A, Nordestgaard BG. C-reactive protein as a predictor of prognosis in chronic obstructive pulmonary disease. Am J Respir Crit Care Med 2007; 175: 250-5.

19. Torres JP, Pinto-Plata V, Casanova C, Mullerova H, Córdoba-Lanús E, Muros de Fuentes $M$, et al. C-reactive protein levels and survival in patients with moderate to very severe COPD. Chest 2008; 133: 1336-43.

20. Gagnon $P$, Guenette JA, Langer D, Laviolette L, Mainguy V, Maltais F, Ribeiro $F$, et al. Pathogenesis of hyperinflation in chronic obstructive pulmonary diseases. Int J Chron Obstruct Pulmon Dis 2014; 9: 187-201.

21. Stolz D, Breidthardt T, Christ-Crain M, Bingisser R, Miedinger D, Leuppi $\mathrm{J}$, et al. Use of B-type natriuretic peptide in the risk stratification of acute exacerbations of COPD. Chest 2008; 133: 1088-94.

22. Eroğlu S, Yildirir A, Bozbas H, Aydinalp A, Ulubay G, Eldem O, et al. Brain natriuretic peptide levels and cardiac functional capacity in patients with dyspnea and isolated diastolic dysfunction. Int Heart J 2007; 48: 97-106.

23. Saryal S. Etiopathogenesis and diagnostic methods in COPD. Thoracic Society I. Winter School 2002: 23-37.

24. Bauerle O, Chrusch CA, Younes M. Mechanisms by which COPD affects exercise tolerance. Am J Respir Crit Care Med 1998; 157: 57-68.

25. Midorikawa J, Hida W, Taguchi O, Okabe S, Kurosawa H, Mizusawa A, pulmonary disease. Respiration 1997; 64: 76-80.

26. Sahebjami H, Sathianpitayakul E. Influence of body weight on the severity of dyspnea in chronic obstructive pulmonary disease. Am J Respir Crit Care Med 2000; 161: 886-90

27. Schols AM, Soeters PB, Mortert R, Pluymers J, Wouters EF. Physiologic effects of nutritional support and anabolic steroids in patients with chronic obstructive pulmonary disease. Am J Respir Crit Care Med 1995; 152: $1268-74$

28. Creutzberg EC, Wouters EFM, Mostert R, Weling-Scheepers CAPM, Schols AMWJ. Efficacy of nutritional supplementation therapy in depleted patients with chronic obstructive pulmonary disease. Nutrition 2003; 19: 120-7. et al. Lack of ventilatory threshold in patients with chronic obstructive 